\title{
Combined Multiuser Detection and Beamforming for CDMA Systems: Filter Structures
}

\author{
Aylin Yener, Member, IEEE, Roy D. Yates, Member, IEEE, and Sennur Ulukus, Member, IEEE
}

\begin{abstract}
Code-division multiple-access (CDMA) systems are interference limited, and therefore efficient interference management is necessary to enhance the capacity of a CDMA system. In this paper, we consider combining two effective receiver-based interference management strategies: multiuser detection (temporal filtering) and receiver beamforming (spatial filtering). We formulate and examine the performance of several two-dimensional linear filter structures, which are all based on minimum mean squared error (MMSE) criterion but differ in how the MMSE problems are defined in the temporal and spatial domains, i.e., jointly or in cascade. It is shown that while the joint optimum MMSE filter achieves the maximum signal-to-interference ratio (SIR) among all possible matrix filters, the constrained optimum MMSE filter which results in a single temporal and single spatial filter, outperforms all combined single-user/multiple-user approaches and cascaded optimization approaches either uniformly or asymptotically. The constrained optimum MMSE filter is near-far resistant in all but very highly loaded systems and enjoys low complexity.
\end{abstract}

Index Terms-CDMA, interference suppression, MMSE, multiuser detection, receiver beamforming.

\section{INTRODUCTION}

$\mathbf{T}$ HE demand for high-capacity flexible wireless services is ever-growing. Code-division multiple access (CDMA) shows promise in meeting this demand, and consequently wireless CDMA (WCDMA) [1]-[3] has been a strong candidate to be a standard for third-generation (3G) wireless systems. It is well known that CDMA systems are interference limited and suffer from near-far effect, where strong users may create excessive interference and degrade the performance of the weak users significantly. The challenge to enhance the capacity of a CDMA system therefore lies in interference management. Many techniques that control and/or suppress interference in CDMA systems by transmit and/or receiver side processing such as transmit power control, multiuser detection, receiver beamforming, precoding using signature selection, and transmit beamforming have been proposed to date; see, for example, [4]-[8]. In this paper, we concentrate on the two commonly used receiver processing-based interference management methods: multiuser detection and receiver beamforming. Both

Manuscript received January 10, 2000; revised July 25, 2001.

A. Yener is with the Electrical Engineering Department, The Pennsylvania State University, University Park, PA 16802 USA (e-mail: yener@ee.psu.edu).

R. D. Yates is with WINLAB, Rutgers-The State University, Piscataway, NJ 08854 USA (e-mail: ryates@winlab.rutgers.edu).

S. Ulukus is with the Department of Electrical and Computer Engineering, University of Maryland, College Park, MD 20742 USA (e-mail ulukus@eng.umd.edu).

Digital Object Identifier 10.1109/TVT.2002.800616 methods aim at suppressing or cancelling the interference using receiver signal processing; multiuser detection exploits the temporal structure, whereas beamforming exploits the spatial structure of the interference.

Multiuser detection [5] performs temporal filtering of the received signal to effectively suppress the multiple-access interference. The optimum multiuser detector has been shown to be exponentially complex in the number of users [9]. A number of low-complexity suboptimum receivers have been proposed following this development [10]-[12]. Among these low-complexity receivers, the minimum mean squared error (MMSE) detector [11] minimizes the expected squared error between the transmitted signal and the output of the receiver filter. Increasing the capacity of CDMA systems by employing antenna arrays at the base station is proposed in [6], where the outputs of the multiple antenna array elements are combined to make bit decisions for the user. Matched filter receivers are assumed in the temporal domain for each user, and the array observations are combined via a filter that is matched to the array response of the user, i.e., single user processing is employed in both domains.

Another method of capacity enhancement that utilizes the spatial diversity is space-time processing for CDMA, which traditionally refers to receiver beamforming (space processing) and multipath combining (time processing) [13]. The received signals from different paths and antennas are combined to better decode the desired user's bits. However, the inherent structure of the multiple-access interference is not exploited, i.e., no multiuser detection is employed [14]-[16]. A recent paper [17] addresses the derivation of the sufficient statistics and the optimum and some linear suboptimum multiuser detectors when an antenna array is present at the receiver for a multipath channel.

In this paper, we will investigate the possible receiver filter structures when both multiuser detection and beamforming are employed to further increase the uplink capacity of a CDMA system. Linear processing is assumed in both the temporal and the spatial domains and the temporal-spatial filters are denoted by two-dimensional matrix filters. Within this framework, there are several possible filter structures. One can derive the jointly optimal temporal and spatial filter that minimizes the mean squared error between the information bit and the filter output of a desired user. Since this joint MMSE filter may have high computational complexity, less complex filters, which nevertheless provide efficient interference suppression, are of interest. To serve this purpose, recently, constrained optimum filters are proposed by forcing the joint receiver filter matrix to be of rank 1 and finding the optimum filter in this constrained space [18], [19]. One can also construct cascaded filters where mean squared error optimization is carried over 
in temporal and spatial domains independently in tandem, in both spatial-temporal and temporal-spatial orders. While the cascaded spatial-temporal filter is similar to the idea of cascade optimum-space/optimum-time combiner proposed in [14], the temporal combiner in our case is a chip combiner that exploits the temporal structure of the interference composed of the temporal signatures of the interferers, as opposed to a multipath combiner in [14], which is a single-user temporal processor. We consider all the above filter structures and then give analytical performance comparisons among them. While the joint domain MMSE filter is clearly the signal-to-interference ratio (SIR) maximizing temporal and spatial processor over all matrix filters, an interesting observation is that the constrained optimum temporal spatial processor outperforms all combined single-user/multiple-user approaches and the cascaded approaches either uniformly or asymptotically. Numerical results supporting the performance analysis are also given.

\section{SYSTEM MODEL}

We consider a single-cell DS-CDMA system where each user is assigned a unique signature sequence. For clarity of exposition, we assume a synchronous system with processing gain $G$. At the base station, an antenna array of $K$ elements is employed. The received signal at the output of the antenna array at the base station is

$$
\mathbf{r}(t)=\sum_{j=1}^{N} \sqrt{p_{j} h_{j}} b_{j} s_{j}(t) \mathbf{a}_{j}+\mathbf{n}(t)
$$

where $p_{j}, b_{j}$, and $s_{j}(t)$ are the transmit power, bit, and signature of user $j$, respectively. $h_{j}$ and $\mathbf{a}_{j}$ are the uplink gain and the array response vector, i.e., the spatial signature, of user $j$. We assume that both the temporal and spatial signatures of the users are of unit energy, i.e., $\int_{0}^{T} s_{j}(t)^{2} d t=\mathbf{a}_{j}^{H} \mathbf{a}_{j}=1$, where $T$ is the bit duration. We further assume that the temporal signatures are of the following form:

$$
s_{j}(t)=\sum_{l=1}^{G} s_{j}^{(l)} \psi\left(t-(l-1) T_{c}\right) \quad \text { with } s_{j}^{(l)}= \pm 1 / \sqrt{G}
$$

where $\psi(t)$ is the chip waveform and $T_{c}$ is the chip duration. By chip matched filtering and sampling the received signal $\mathbf{r}(t)$ at the $l$ th chip interval, we obtain a $K$-dimensional observation vector $\mathbf{r}_{l}$. The vector $\mathbf{r}_{l}$ represents the chip matched filtered samples at the $l$ th chip interval across the $K$ antenna array elements and is given by

$$
\mathbf{r}_{l}=\sum_{j=1}^{N} \sqrt{p_{j} h_{j}} b_{j} \mathbf{a}_{j} s_{j}^{(l)}+\mathbf{n}_{l} .
$$

Over one bit period $T=G T_{c}$, we collect a set of $G K$-dimensional vectors $\left\{\mathbf{r}_{l}, 1 \leq l \leq G\right\}$, which we can arrange in a $G \times K$ matrix $\mathbf{R}=\left[\mathbf{r}_{1}, \mathbf{r}_{2}, \ldots, \mathbf{r}_{G}\right]^{\top}$; see Fig. 1 .

$$
\mathbf{R}=\sum_{j=1}^{N} \sqrt{p_{j} h_{j}} b_{j} \mathbf{s}_{j} \mathbf{a}_{j}^{\top}+\mathbf{N} .
$$

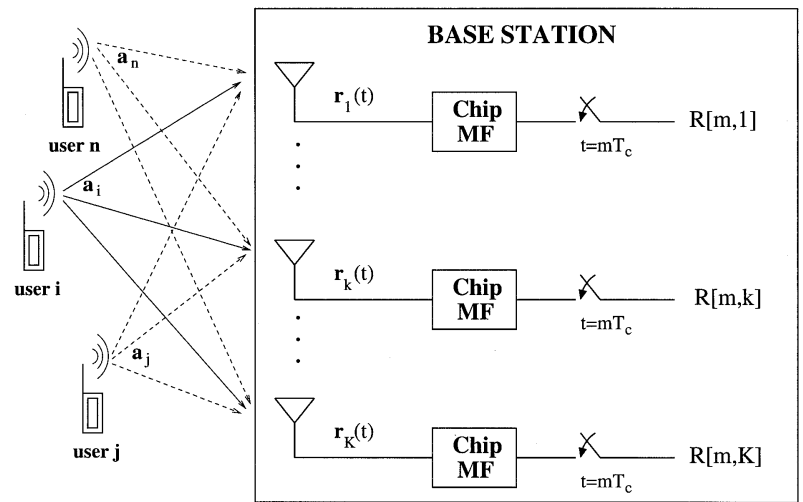

Fig. 1. Received signal model at the base station, $R[m, k]$ denotes the $(m, k)$ th element of the received signal matrix $\mathbf{R}$ given in (4).

These $K G$ observation samples can also be seen as a collection of $K G$-dimensional vectors, i.e., $\mathbf{R}=\left[\mathbf{z}_{1}, \mathbf{z}_{2}, \ldots, \mathbf{z}_{K}\right]$, where $\mathbf{z}_{k}$ denotes the observation vector consisting $G$ chip matched filtered samples at the output of the $k$ th antenna element and is expressed as

$$
\mathbf{z}_{k}=\sum_{j=1}^{N} \sqrt{p_{j} h_{j}} b_{j} \mathbf{s}_{j} a_{j}^{(k)}+\mathbf{n}_{k} .
$$

In (4), $\mathbf{N}$ is the matrix that represents the spatially and temporally white noise, i.e., $E\left[N_{k l}^{*} N_{m n}\right]=\sigma^{2} \delta_{k m} \delta_{l n}$, where $(\cdot)^{*}$ denotes the conjugate of a complex number. We label user $i$ as the desired user and the other users as interferers. The rest of this paper deals with designing temporal-spatial filters for user $i$.

\section{Filter Structures}

The detection of the information bit of the desired user, which we will assume to be user $i$ in the sequel, is done by taking the sign of the real part of the decision statistic, which is found by combining the entries of the observation matrix $\mathbf{R}$ by using a matrix filter $\mathbf{X}_{i}$. Thus, the decision statistic $y_{i}$ is the output of a two-dimensional linear filter $\mathbf{X}_{i}$

$$
y_{i}=\sum_{j=1}^{G} \sum_{l=1}^{K}\left[X_{i}\right]_{j l}^{*} R_{j l}=\operatorname{tr}\left(\mathbf{X}_{i}^{H} \mathbf{R}\right)
$$

where $\operatorname{tr}(\cdot)$ and $(\cdot)^{H}$ are the trace and the hermitian transpose operations on a matrix, respectively. The representation of the temporal-spatial filter as a matrix is useful in expressing any filtering scheme that is linear in the temporal and spatial domains.

In what follows, we investigate the possible filter structures. The filter structures in Section III-A-C use single-user processing in at least one of the temporal and spatial domains and are well-known. Next we derive two temporal-spatial filters, the filter structures in Section III-D and III-E, which use cascaded MMSE optimizations in spatial and temporal domains. The structure of these receivers is to combine either the received chip samples at the output of each array element in the MMSE sense followed by a spatial filter that combines the resulting vector in the MMSE sense (Section III-D); or to combine all array outputs for each chip sample in the MMSE sense 
followed by the temporal MMSE combiner for the resulting vector (Section III-E).

Next, in Section III-F and III-G, we give the joint temporal-spatial MMSE filter structures. The difference between the two joint MMSE structures is the fact that while the joint optimum temporal-spatial MMSE in Section III-F is the best filter in terms of minimizing the mean squared error (and maximizing the SIR) over all possible matrix filters, the constrained optimum temporal-spatial filter in Section III-G is the MMSE filter when the filter space is constrained to contain matrix filters of rank 1 only. The physical interpretation of this mathematical constraint on the matrix receiver filter is that it results in a separable filter with a single temporal and a single spatial combiner.

The difference between the joint MMSE structures in Section III-F and III-G and the cascaded MMSE structures in Section III-D and III-E lies in the fact that the cascaded structures use temporal and spatial filters that are optimized independently in each domain while the joint structures are found by optimization in both domains simultaneously.

\section{A. Single-User Temporal-Spatial Detector}

This is a single-user-based approach for both the spatial and the temporal domains and has been proposed in [6]. The decision statistic in this case is $y_{i}=\mathbf{s}_{i}^{\top} \mathbf{R} \mathbf{a}_{i}^{*}=\operatorname{tr}\left(\mathbf{a}_{i}^{*} \mathbf{s}_{i}^{\top} \mathbf{R}\right)$, leading to

$$
\mathbf{X}_{i}^{\mathrm{MF}-\mathrm{MF}}=\mathbf{s}_{i} \mathbf{a}_{i}^{\top}
$$

\section{B. Temporal MMSE Filter-Spatial Matched Filter}

This approach uses multiuser processing in temporal domain [11] and single-user processing in spatial domain. The decision statistic in this case is $y_{i}=\mathbf{c}_{i}^{\top} \mathbf{R a}_{i}^{*}=\operatorname{tr}\left(\mathbf{a}_{i}^{*} \mathbf{c}_{i}^{\top} \mathbf{R}\right)$, leading to

$$
\mathbf{X}_{i}^{\mathrm{MMSE}-\mathrm{MF}}=\mathbf{c}_{i} \mathbf{a}_{i}^{\top}
$$

where

$$
\mathbf{c}_{i}=\sqrt{p_{i} h_{i}}\left(\sum_{j=1}^{N} p_{j} h_{j}\left|\mathbf{a}_{i}^{H} \mathbf{a}_{j}\right|^{2} \mathbf{s}_{j} \mathbf{s}_{j}^{\top}+\sigma^{2} \mathbf{I}\right)^{-1} \mathbf{s}_{i} .
$$

\section{Temporal Matched Filter-Spatial MMSE Filter}

This approach uses single-user processing in the temporal domain combined with multiuser processing in spatial domain [20]. The decision statistic in this case is $y_{i}=\mathbf{s}_{i}^{\top} \mathbf{R} \mathbf{w}_{i}^{*}=$ $\operatorname{tr}\left(\mathbf{w}_{i}^{*} \mathbf{s}_{i}^{\top} \mathbf{R}\right)$, leading to

$$
\mathbf{X}_{i}^{\mathrm{MF}-\mathrm{MMSE}}=\mathbf{s}_{i} \mathbf{w}_{i}^{\top}
$$

where

$$
\mathbf{w}_{i}=\sqrt{p_{i} h_{i}}\left(\sum_{j=1}^{N} p_{j} h_{j}\left(\mathbf{s}_{i}^{\top} \mathbf{s}_{j}\right)^{2} \mathbf{a}_{j} \mathbf{a}_{j}^{H}+\sigma^{2} \mathbf{I}\right)^{-1} \mathbf{a}_{i}
$$

\section{Cascaded Temporal-Spatial MMSE}

Assume that at the output of each antenna array, we are allowed to design a temporal filter, i.e., a linear chip combiner. Recall that the output of the $k$ th element of the antenna array corresponds to the $k$ th column, $\mathbf{z}_{k}$, of the received signal matrix $\mathbf{R}$ and is given by (5).

We would like to design $K$ temporal filters $\mathbf{c}_{k}, k=1, \ldots, K$, such that the resulting statistic

$$
\tilde{y}_{k}=\mathbf{c}_{k}^{H} \mathbf{z}_{k}=\sum_{j=1}^{N} \sqrt{p_{j} h_{j}} b_{j} \mathbf{c}_{k}^{H} \mathbf{s}_{j} a_{j}^{(k)}+\mathbf{c}_{k}^{H} \mathbf{n}_{k}
$$

has minimum mean squared difference from the desired bit $b_{i}$. The solution can be found as [11]

$$
\begin{aligned}
\mathbf{c}_{k} & =\arg \min _{\mathbf{c}} E\left[\left|\mathbf{c}^{H} \mathbf{z}_{k}-b_{i}\right|^{2}\right] \\
& =\sqrt{p_{i} h_{i}} a_{i}^{(k)}\left(\sum_{j=1}^{N}\left|a_{j}^{(k)}\right|^{2} p_{j} h_{j} \mathbf{s}_{j} \mathbf{s}_{j}^{\top}+\sigma^{2} \mathbf{I}\right)^{-1} \mathbf{s}_{i} .
\end{aligned}
$$

Note that what make $\mathbf{c}_{k}$, the temporal MMSE filter at the output of the $k$ th antenna, different from $\mathbf{c}_{l}$, the temporal MMSE filter at the output of the $l$ th antenna, are the different spatial gains users have to different antennas. Defining the modified gain at the output of the $k$ th antenna for user $j$ as $\left(\mathbf{c}_{k}^{H} \mathbf{s}_{j}\right) a_{j}^{(k)}$, from (12), we have

$$
\tilde{y}_{k}=\sum_{j=1}^{N} \sqrt{p_{j} h_{j}} b_{j} \tilde{a}_{j}^{(k)}+\tilde{n}_{k}, \quad k=1, \ldots, K
$$

with

$$
\begin{aligned}
\tilde{a}_{j}^{(k)}= & \left(\sqrt{p_{i} h_{i}}\left(a_{i}^{(k)}\right)^{*} \mathbf{s}_{i}^{\top}\right. \\
& \left.\times\left(\sum_{j^{\prime}=1}^{N}\left|a_{j^{\prime}}^{(k)}\right|^{2} p_{j} h_{j^{\prime}} \mathbf{s}_{j^{\prime}} \mathbf{s}_{j^{\prime}}^{\top}+\sigma^{2} \mathbf{I}\right)^{-1} \mathbf{s}_{j}\right) a_{j}^{(k)} .
\end{aligned}
$$

We can then combine $\left\{\tilde{y}_{k}\right\}$ in the MMSE sense. Similar to (13), we can express the second stage of the cascaded filter as

$$
\begin{aligned}
\mathbf{w} & =\arg \min _{\mathbf{w}} E\left[\left|\mathbf{w}^{H} \tilde{\mathbf{y}}-b_{i}\right|^{2}\right] \\
& =\sqrt{p_{i} h_{i}}\left(\sum_{j=1}^{N} p_{j} h_{j} \tilde{\mathbf{a}}_{j} \tilde{\mathbf{a}}_{j}^{H}+\boldsymbol{\Lambda}\right)^{-1} \tilde{\mathbf{a}}_{i}
\end{aligned}
$$

where $\boldsymbol{\Lambda}=\operatorname{diag}\left\{\sigma^{2}\left(\mathbf{c}_{k}^{H} \mathbf{c}_{k}\right)\right\}$ is the covariance matrix of $\tilde{\mathbf{n}}$. The final bit decision is done by taking the sign of the real part of $y_{i}=\mathbf{w}^{H} \tilde{\mathbf{y}}$.

Note that to construct the overall receiver, we need to invert $K G \times G$ matrices and one $K \times K$ matrix. To see how the overall 
cascaded filter can be expressed as a matrix filter $\mathbf{X}_{i}^{\mathrm{TS}-\mathrm{CMMSE}}$, observe that

$$
\begin{aligned}
\tilde{y}_{k} & =\mathbf{c}_{k}^{H} \mathbf{z}_{k}=\mathbf{c}_{k}^{H} \mathbf{R e}_{k}=\operatorname{tr}\left(\mathbf{e}_{k} \mathbf{c}_{k}^{H} \mathbf{R}\right) \\
\text { and } & \\
y_{i} & =\sum_{k=1}^{K} w_{k}^{*} \tilde{y}_{k}=\sum_{k=1}^{K} w_{k}^{*} \operatorname{tr}\left(\mathbf{e}_{k} \mathbf{c}_{k}^{H} \mathbf{R}\right) \\
& =\operatorname{tr}\left(\sum_{k=1}^{K} w_{k}^{*} \mathbf{e}_{k} \mathbf{c}_{k}^{H} \mathbf{R}\right)
\end{aligned}
$$

where $\mathbf{e}_{k}$ is a $K$ vector which has a one in its $k$ th entry and all zeros elsewhere. Comparing (18) with (6), we find that

$$
\mathbf{X}_{i}^{\mathrm{TS}-\mathrm{CMMSE}}=\sum_{k=1}^{K} w_{k} \mathbf{c}_{k} \mathbf{e}_{k}^{\top}
$$

which can be of rank up to $K$.

\section{E. Cascaded Spatial-Temporal MMSE}

Alternatively, one can think of first combining all antenna array elements for each chip sample, followed by a temporal combiner. In this case, first, each row of the received matrix $\mathbf{r}_{l}$ from (3) is combined via a spatial filter $\mathbf{w}_{l}, l=1, \ldots, G$.

We would like to find the filter $\mathbf{w}_{l}$ to combine the elements of $\mathbf{r}_{l}$ in the MMSE sense. The resulting filter can be expressed as

$$
\begin{aligned}
\mathbf{w}_{l} & =\arg \min _{\mathbf{w}} E\left[\left|\mathbf{w}^{H} \mathbf{r}_{l}-b_{i}\right|^{2}\right] \\
& =\sqrt{p_{i} h_{i}} s_{i}^{(l)}\left(\sum_{j=1}^{N}\left(s_{j}^{(l)}\right)^{2} p_{j} h_{j} \mathbf{a}_{j} \mathbf{a}_{j}^{H}+\sigma^{2} \mathbf{I}\right)^{-1} \mathbf{a}_{i} .
\end{aligned}
$$

Recall from (2) that $\left(s_{j}^{(l)}\right)^{2}=1 / G$, for all $l$. Thus, defining

$$
\hat{\mathbf{w}}=\sqrt{p_{i} h_{i}}\left(\sum_{j=1}^{N} \frac{1}{G} p_{j} h_{j} \mathbf{a}_{j} \mathbf{a}_{j}^{H}+\sigma^{2} \mathbf{I}\right)^{-1} \mathbf{a}_{i}
$$

we arrive at

$$
\mathbf{w}_{l}=s_{i}^{(l)} \hat{\mathbf{w}} \quad l=1, \ldots, G .
$$

Thus, for each chip sample sequence, $\mathbf{w}_{l}$ is equal to $\pm \hat{\mathbf{w}}$ depending on the current chip value of the desired user. At the output of the lth combiner, the resulting statistic can be expressed as

$$
\begin{aligned}
\hat{y_{l}} & =\mathbf{w}_{l}^{H} \mathbf{r}_{l}=\sum_{j=1}^{N} \sqrt{p_{j} h_{j}} b_{j} \mathbf{w}_{l}^{H} \mathbf{a}_{j} s_{j}^{(l)}+\mathbf{w}_{l}^{H} \mathbf{n}_{l} \\
& =\sum_{j=1}^{N} \sqrt{p_{j} h_{j}} b_{j} \hat{\mathbf{w}}^{H} \mathbf{a}_{j} s_{i}^{(l)} s_{j}^{(l)}+\hat{n}_{l} .
\end{aligned}
$$

Defining

$$
\hat{s}_{j}^{(l)}=\hat{\mathbf{w}}^{H} \mathbf{a}_{j} s_{i}^{(l)} s_{j}^{(l)}
$$

we have

$$
\hat{y}_{l}=\sum_{j=1}^{N} \sqrt{p_{j} h_{j}} b_{j} \hat{s}_{j}^{(l)}+\hat{n}_{l}, \quad l=1, \ldots, G .
$$

It remains to find the MMSE combiner for $\hat{\mathbf{y}}$. We can express this second stage of the cascaded filter as

$$
\begin{aligned}
\mathbf{c} & =\arg \min _{\mathbf{c}} E\left[\left|\mathbf{c}^{H} \hat{\mathbf{y}}-b_{i}\right|^{2}\right] \\
& =\sqrt{p_{i} h_{i}}\left(\sum_{j=1}^{N} p_{j} h_{j} \hat{\mathbf{s}}_{j} \hat{\mathbf{s}}_{j}^{H}+\frac{1}{G} \sigma^{2}\left(\hat{\mathbf{w}}^{H} \hat{\mathbf{w}}\right) \mathbf{I}\right)^{-1} \hat{\mathbf{s}}_{i} .
\end{aligned}
$$

Notice that, as in the case of Section III-D, the noise covariance matrix is given by $\operatorname{diag}\left\{\sigma^{2}\left(\mathbf{w}_{l}^{T} \mathbf{w}_{l}\right)\right\}$. Since $\mathbf{w}_{l}^{H} \mathbf{w}_{l}=(1 / G)\left(\hat{\mathbf{w}}^{H} \hat{\mathbf{w}}\right)$ for all $l$, the noise covariance matrix reduces to $\left(\sigma^{2} / G\right)\left(\hat{\mathbf{w}}^{H} \hat{\mathbf{w}}\right) \mathbf{I}$. The final bit decision is done by taking the sign of the real part of $y_{i}=\mathbf{c}^{H} \hat{\mathbf{y}}$.

Note that to construct the overall receiver, we need to invert one $K \times K$ matrix to calculate $\hat{\mathbf{w}}$ and one $G \times G$ matrix to calculate $\mathbf{c}$. To see how the overall cascaded filter can be expressed as a matrix filter $\mathbf{X}_{i}^{\mathrm{ST}-\mathrm{CMMSE}}$, observe that

$$
\begin{aligned}
\hat{y}_{l} & =\mathbf{e}_{l}^{\top} \mathbf{R} \mathbf{w}_{l}^{*}=s_{i}^{(l)} \operatorname{tr}\left(\hat{\mathbf{w}}^{*} \mathbf{e}_{l}^{\top} \mathbf{R}\right) \\
y_{i} & =\mathbf{c}^{H} \hat{\mathbf{y}}=\sum_{l=1}^{G} c_{l}^{*} \hat{y}_{l}=\sum_{l=1}^{G} c_{l}^{*} s_{i}^{(l)} \operatorname{tr}\left(\hat{\mathbf{w}}^{*} \mathbf{e}_{l}^{\top} \mathbf{R}\right) \\
& =\operatorname{tr}\left(\hat{\mathbf{w}}^{*}\left(\sum_{l=1}^{G} c_{l}^{*} s_{i}^{(l)} \mathbf{e}_{l}^{\top}\right) \mathbf{R}\right) .
\end{aligned}
$$

Then, comparing (29) with (6), we find that

$$
\mathbf{X}_{i}^{\mathrm{ST}-\mathrm{CMMSE}}=\left(\sum_{l=1}^{G} c_{l} s_{i}^{(l)} \mathbf{e}_{l}\right) \hat{\mathbf{w}}^{\top} .
$$

\section{F. Optimum Temporal-Spatial MMSE Filter}

Consider the optimum matrix filter in temporal and spatial domains, which minimizes the minimum mean squared error between $y_{i}$ and $b_{i}$

$$
\mathbf{X}_{i}^{\mathrm{O}-\mathrm{MMSE}}=\arg \min _{\mathbf{X}} E\left[\left|\operatorname{tr}\left(\mathbf{X}^{H} \mathbf{R}\right)-b_{i}\right|^{2}\right] .
$$

The optimization problem (31) can be converted to an optimization problem with vector variables for easier manipulation [21]. The problem to solve then becomes a straightforward extension of the temporal or spatial (single-domain) MMSE problem and can easily be found as [11], [17], [19]-[22]

$$
\mathbf{x}_{i}=\sqrt{p_{i} h_{i}}\left(\sum_{j=1}^{N} p_{j} h_{j} \mathbf{q}_{j} \mathbf{q}_{j}^{H}+\sigma^{2} \mathbf{I}\right)^{-1} \mathbf{q}_{i}
$$

where $\mathbf{q}_{j}$ is the combined temporal-spatial signature of user $j$ and is constructed by stacking columns of $\mathbf{s}_{j} \mathbf{a}_{j}^{\top}$ as a long vector of size $K G$. The matrix filter $\mathbf{X}_{i}^{\mathrm{O}-\mathrm{MMSE}}$ is constructed by taking every $G$ elements of $\mathbf{x}_{i}$ and putting as a column to $\mathrm{X}_{i}^{\mathrm{O}-\mathrm{MMSE}}$.

The joint MMSE filter requires a possibly large matrix $(K G \times K G)$ to be inverted, which can be computationally costly, or the corresponding adaptive implementation may be 
slow. This is the reason why we consider the less complex joint MMSE filter in the next section.

\section{G. Constrained Optimum Temporal-Spatial MMSE Filter}

To reduce the complexity of the temporal and spatial filtering with little sacrifice in performance, [18] proposes finding the optimum matrix filter in a constrained class of matrix filters. The proposed constrained class is rank 1 matrix filters, or the separable temporal-spatial filters, i.e., the filters that can be expressed as $\mathbf{X}_{i}=\mathbf{c w}^{\dagger}$. We can find the joint optimal filter pair in the minimum mean squared error sense for this constrained class. The optimization problem in (31) becomes

$$
\begin{aligned}
{[\overline{\mathbf{c}}, \overline{\mathbf{w}}] } & =\arg \min _{\mathbf{c}, \mathbf{w}} E\left[\left|\operatorname{tr}\left(\mathbf{w}^{*} \mathbf{c}^{H} \mathbf{R}\right)-b_{i}\right|^{2}\right] \\
& =\arg \min _{\mathbf{c}, \mathbf{w}} E\left[\left|\mathbf{c}^{H} \mathbf{R} \mathbf{w}^{*}-b_{i}\right|^{2}\right] .
\end{aligned}
$$

The resulting $[\overline{\mathbf{c}}, \overline{\mathbf{w}}]$ pair yields the matrix filter

$$
\mathbf{X}_{i}^{\mathrm{CO}-\mathrm{MMSE}}=\overline{\mathbf{c}}^{\top}{ }^{\top} \text {. }
$$

This matrix filter is suboptimal for the optimization problem given in (31) since it is found in a constrained $\mathbf{X}_{i}$ space. The MSE function in (33) can be expressed as

$$
\begin{aligned}
\mathrm{MSE}= & \sum_{j=1}^{N} p_{j} h_{j}\left|\mathbf{c}^{H} \mathbf{s}_{j}\right|^{2}\left|\mathbf{w}^{H} \mathbf{a}_{j}\right|^{2}+\sigma^{2}\left(\mathbf{c}^{H} \mathbf{c}\right)\left(\mathbf{w}^{H} \mathbf{w}\right) \\
& -2 \sqrt{p_{i} h_{i}} \Re\left\{\left(\mathbf{c}^{H} \mathbf{s}_{i}\right)\left(\mathbf{w}^{H} \mathbf{a}_{i}\right)\right\}+1
\end{aligned}
$$

where $\Re\{\cdot\}$ denotes the real part of a complex number. The minimizer of (35) does not have a closed-form expression [19]. Further, the MSE function is not jointly convex in $\mathbf{c}$ and $\mathbf{w}$, although it is convex in each variable (c, or w), when the other variable is fixed. Thus, standard iterative optimization algorithms cannot guarantee convergence to global minima. However, an alternating minimization algorithm [23] is given in [19] that is observed to have good convergence properties. We restate the algorithm here for convenience.

Consider fixing the value of one of the filters; say $\mathbf{w}$ is fixed to $\tilde{\mathbf{w}}$. It is then possible to find the filter $\hat{\mathbf{c}}$ that maximally decreases the MSE function in (35). The solution is analogous to the MMSE detector [11], where user $j$ 's received amplitude is modified such that it is $\sqrt{p_{j} h_{j}}\left(\tilde{\mathbf{w}}^{H} \mathbf{a}_{j}\right)$. Denote this filter $\hat{\mathbf{c}}=\operatorname{MMSE}(\tilde{\mathbf{w}})$

$$
\begin{aligned}
\hat{\mathbf{c}}= & \operatorname{MMSE}(\tilde{\mathbf{w}}) \\
= & \sqrt{p_{i} h_{i}}\left(\tilde{\mathbf{w}}^{H} \mathbf{a}_{i}\right) \\
& \times\left(\sum_{j=1}^{N} p_{j} h_{j}\left|\tilde{\mathbf{w}}^{H} \mathbf{a}_{j}\right|^{2} \mathbf{s}_{j} \mathbf{s}_{j}^{H}+\sigma^{2}\left(\tilde{\mathbf{w}}^{H} \tilde{\mathbf{w}}\right) \mathbf{I}\right)^{-1} \mathbf{s}_{i} .
\end{aligned}
$$

The same argument can be made for the case where $\mathbf{c}$ is fixed to $\tilde{\mathbf{c}}$ and the spatial filter is found to maximally decrease the MSE, $\hat{\mathbf{w}}=\operatorname{MMSE}(\tilde{\mathbf{c}})$

$$
\begin{aligned}
\hat{\mathbf{w}}= & \operatorname{MMSE}(\tilde{\mathbf{c}}) \\
= & \sqrt{p_{i} h_{i}}\left(\tilde{\mathbf{c}}^{H} \mathbf{s}_{i}\right) \\
& \times\left(\sum_{j=1}^{N} p_{j} h_{j}\left|\tilde{\mathbf{c}}^{H} \mathbf{s}_{j}\right|^{2} \mathbf{a}_{j} \mathbf{a}_{j}^{H}+\sigma^{2}\left(\tilde{\mathbf{c}}^{H} \tilde{\mathbf{c}}\right) \mathbf{I}\right)^{-1} \mathbf{a}_{i} .
\end{aligned}
$$

Now, consider the following algorithm. Starting with the filter pair $\mathbf{c}(0), \mathbf{w}(0)$ and keeping $\mathbf{w}(0)$ fixed, one can find $\mathbf{c}(1)=\operatorname{MMSE}(\mathbf{w}(0))$. This operation decreases the MSE defined in (35). Then keeping $\mathbf{c}(1)$ fixed, one can find $\mathbf{w}(1)=\operatorname{MMSE}(\mathbf{c}(1))$. This operation further decreases the MSE in (35). Iteration $n+1$ of this two-step iterative algorithm for user $i$ is given as

$$
\begin{aligned}
\mathbf{c}(n+1) & =\operatorname{MMSE}(\mathbf{w}(n)) \\
\mathbf{w}(n+1) & =\operatorname{MMSE}(\mathbf{c}(n+1))
\end{aligned}
$$

Note that the order in which $\mathbf{c}$ and $\mathbf{w}$ are updated could be reversed, i.e., we could devise the same algorithm where the $\mathbf{w}$ vector is updated before $\mathbf{c}$. After each two-step iteration given by (38) and (39), the MSE in (35) monotonically decreases. The algorithm is provably convergent, and the convergence point is experimentally observed to be the optimum pair $[\overline{\mathbf{c}}, \overline{\mathbf{w}}]$, where the MSE is minimized and the SIR of the user is maximized [19].

\section{PERformance COMPARISON}

An important performance comparison criterion is the probability of bit error. Unfortunately, for general system parameters, it is difficult to derive analytical results for the probability of bit error, rendering this comparison intractable. A commonly used approximation to the probability of bit error is obtained when a Gaussian approximation is applied to the total interference. It was reported in [24] that this Gaussian approximation is particularly accurate when MMSE receivers are employed. In this case, the bit error probability becomes a one-to-one decreasing function of the SIR, which, in turn, can be related to the MSE. For a general matrix filter $\mathbf{X}$, the MSE and the SIR are related as (for details, see [19, Appendix])

$$
\frac{1}{\min _{\alpha} \operatorname{MSE}(\alpha \mathbf{X})}=1+\operatorname{SIR}(\mathbf{X}) .
$$

Thus, with an appropriate scaling, the MSE and the SIR produced by every filter can be related, and the filter that minimizes the MSE also maximizes the SIR. Note that the SIR, and therefore the bit error rate when defined in terms of the SIR, are insensitive to the scaling of the linear receiver filter.

From the arguments above, it is clear that the optimum MMSE receiver of Section III-F outperforms all other receiver structures mentioned in Section III-A-E, as well as the constrained optimum MMSE receiver in Section III-G, in terms of both the MSE and the SIR. The reason for this is that the filter in Section III-F is chosen to minimize the MSE over all possible matrix filters. It only remains to compare the performance of the constrained optimum MMSE receiver of Section III-G with the receiver structures in Section III-A-E. First we observe from (7), (8), (10), and (30) that the filters $\quad \mathbf{X}_{i}^{\mathrm{MF}-\mathrm{MF}}, \mathbf{X}_{i}^{\mathrm{MMSE}-\mathrm{MF}}, \mathbf{X}_{i}^{\mathrm{MF}-\mathrm{MMSE}}, \mathbf{X}_{i}^{\mathrm{ST}-\mathrm{CMMSE}}$ are of rank 1. Given that the constrained optimum MMSE $\mathbf{X}_{i}^{\mathrm{CO}-\mathrm{MMSE}}$ minimizes the MSE and therefore maximizes the SIR among all possible rank 1 matrix filters, we conclude that the constrained optimum MMSE receiver filter outperforms all of these suboptimum receiver filters. In fact, the iterative algorithm described by (38) and (39) can be 
started at any of the temporal-spatial filter pairs that define $\mathbf{X}_{i}^{\mathrm{MF}-\mathrm{MF}}, \mathbf{X}_{i}^{\mathrm{MMSE}-\mathrm{MF}}, \mathbf{X}_{i}^{\mathrm{MF}-\mathrm{MMSE}}$ or $\mathbf{X}_{i}^{\mathrm{ST}-\mathrm{CMMSE}}$. Since each iteration increases the SIR and decreases the MSE monotonically, with each iteration, the performance of the resulting filter pair is better than the previous one and the convergence point temporal-spatial filter pair $\mathbf{X}_{i}^{\mathrm{CO}-\mathrm{MMSE}}$ outperforms the starting-point filter pair.

The cascaded temporal-spatial MMSE filter $\mathbf{X}_{i}^{\mathrm{TS}-\mathrm{CMMSE}}$ in Section III-D can have rank up to $K$, just as the joint optimum MMSE filter of Section III-F, which can have rank up to $\min \{K, G\}$. Thus, there could be cases under which $\mathbf{X}_{i}^{\mathrm{TS}-\mathrm{CMMSE}}$ performs better than the constrained optimum filter; see Section V. However, the fact that $\mathbf{X}_{i}^{\mathrm{TS}-\mathrm{CMMSE}}$ has higher rank than $\mathbf{X}_{i}^{\mathrm{CO}-\mathrm{MMSE}}$ does not necessarily guarantee that it yields a lower MSE or a higher SIR than $\mathbf{X}_{i}^{\mathrm{CO}-\mathrm{MMSE}}$. This is also demonstrated in Section $\mathrm{V}$ and is intuitively pleasing since $\mathbf{X}_{i}^{\mathrm{TS}-\mathrm{CMMSE}}$, in contrast with $\mathbf{X}_{i}^{\mathrm{CO}-\mathrm{MMSE}}$, is not designed with the objective of minimizing the end-to-end MSE of the user; i.e., from the transmitted bit of the $i$ th user to the final filter output of the $i$ th user. Thus, neither $\mathbf{X}_{i}^{C O-M M S E}$ nor $\mathbf{X}_{i}^{\mathrm{TS}-\mathrm{CMMSE}}$ performs uniformly better than the other; depending on the system parameters $(N, G, K$, spatial and temporal signatures of the users, etc.), one may outperform the other.

It is possible to compare the two filters in the asymptotic case when the background noise $\sigma^{2}$ goes to zero, or, equivalently, the received powers of the interfering users go to infinity. It is well known that the MMSE receiver reduces to a decorrelating receiver as the background noise power goes to zero or the received powers of the interfering users go to infinity [5]. The decorrelating receiver [10] is a linear multiuser detector that suppresses the multiaccess interference totally while enhancing the power of the background Gaussian noise. The multiaccess interference is suppressed by projecting the desired user's signal onto the subspace that is orthogonal to the signal space spanned by the interfering users. The decorrelation operation is independent of the received powers of the users and only depends on the signature sequences of the users. The multiaccess interference is suppressed totally if the desired user's signature sequence is linearly independent of the interfering signatures. The temporal decorrelating filter for user $i \mathbf{d}_{i}$ is found by

$$
\mathbf{d}_{i}=\mathbf{S}\left(\mathbf{S}^{H} \mathbf{S}\right)^{-1} \mathbf{e}_{i}=\mathbf{S} \boldsymbol{\Gamma}^{-1} \mathbf{e}_{i}
$$

for a chip matched filtered CDMA received signal where users' temporal signatures $\mathbf{s}_{j}$ constitute the columns of the signature matrix $\mathbf{S}$. Note that $\boldsymbol{\Gamma}$ is the cross-correlation matrix of users' temporal signatures and is invertible if users' signatures are linearly independent. When this is not the case, the decorrelator is found by using the Moore-Penrose generalized inverse of the cross-correlation matrix $\boldsymbol{\Gamma}^{+}$and remains independent of the received powers of the users [10].

Recall now that in the calculation of the cascaded temporalspatial MMSE receiver filter $\mathbf{X}_{i}^{\mathrm{TS}-\mathrm{CMMSE}}$, first, $K$ temporal MMSE receiver filters are found [see (13)] and that these $K$ MMSE receivers, $\mathbf{c}_{k} \mathrm{~s}$, are different due to the fact that the received powers of the users are different at each antenna array element. This is because the actual received powers of the users are multiplied with the square magnitudes of the antenna gains $\left|a_{j}^{(k)}\right|$ at the outputs of different antenna elements. Since in the interference-limited regime where the ratio of the background noise power to received interfering powers goes to zero, the MMSE receivers go to decorrelators, and since decorrelators are independent of the received powers of the users, all $K$ temporal receiver filters become identical, i.e., $\mathbf{c}_{k}=\mathbf{c}$ for all $k$. Note that this is true even when the cross-correlation matrix is not invertible and the Moore-Penrose generalized inverse is used. When the temporal filters at the output of all antenna array elements are the same, the receiver filter $\mathbf{X}_{i}^{\mathrm{TS}-\mathrm{CMMSE}}$ becomes a rank 1 filter. The MSE achieved by $\mathbf{X}_{i}^{\mathrm{TS}-\mathrm{CMMSE}}, \mathrm{MSE}_{i}^{\mathrm{TS}-\mathrm{CMMSE}}$ is larger than that of the constrained optimum MMSE filter $\mathrm{MSE}_{i}^{\mathrm{CO}-\mathrm{MMSE}}$, simply because $\mathbf{X}_{i}^{\mathrm{CO}-\mathrm{MMSE}}$ is the filter that yields the minimum MSE among all rank 1 matrix filters. Thus

$$
\lim _{\sigma^{2} \rightarrow 0} \mathrm{MSE}_{i}^{\mathrm{TS}-\mathrm{CMMSE}} \geq \lim _{\sigma^{2} \rightarrow 0} \mathrm{MSE}_{i}^{\mathrm{CO}-\mathrm{MMSE}} .
$$

Equivalently, using (40), the SIRs achieved by these two filters in this interference-limited regime are compared as

$$
\lim _{\sigma^{2} \rightarrow 0} \operatorname{SIR}_{i}^{\mathrm{TS}-\mathrm{CMMSE}} \leq \lim _{\sigma^{2} \rightarrow 0} \mathrm{SIR}_{i}^{\mathrm{CO}-\mathrm{MMSE}} .
$$

Hence, the constrained optimum MMSE filter outperforms the cascaded temporal-spatial MMSE filter of Section III-D asymptotically.

\section{RESULTS}

Numerical results showing the performance of the filters considered in this paper are now given. We consider a single-cell CDMA system, the base station of which employs a linear antenna array [17]. For each experiment we present here, the temporal signatures and users' positions, which in turn determine the spatial signatures, are created randomly but then kept fixed for that experiment. The experiments are intended to demonstrate the behavior and performance of all the filters under different system loading conditions. For each experiment, we plot the output SIR for the desired user (in linear scale) versus the received signal-to-noise ratios (SNRs) of all interferers (in $\mathrm{dB}$ scale). The severe near-far conditions where the interferers' powers are very high are intended to show the asymptotic behavior of the filters, i.e., their performance in the interference-limited environment where noise level becomes negligible compared to the interference power. The desired user's SNR is kept at $10 \mathrm{~dB}$.

Consider first a system with processing gain $G=8, K=2$ array elements, and $N=2$ users. Fig. 2 shows the output SIR of the desired user. As expected, with only a single interferer present, all filters perform well even under very severe near-far conditions, where the interferer's power is as much as $60 \mathrm{~dB}$ above the desired user's. The only exception is the temporal-spatial matched filter, which is well known to be not near-far resistant. The more interesting observation about this system is better observed in Fig. 3. Recall that we concluded in Section IV that the constrained optimum MMSE filter does not necessarily outperform the cascaded temporal-spatial MMSE filter of Section III-D; it only is as good as or better than the cascaded temporal-spatial MMSE filter asymptotically. Indeed, in Fig. 3, we see that the cascaded temporal-spatial MMSE outperforms the constrained optimum MMSE filter. When the system 


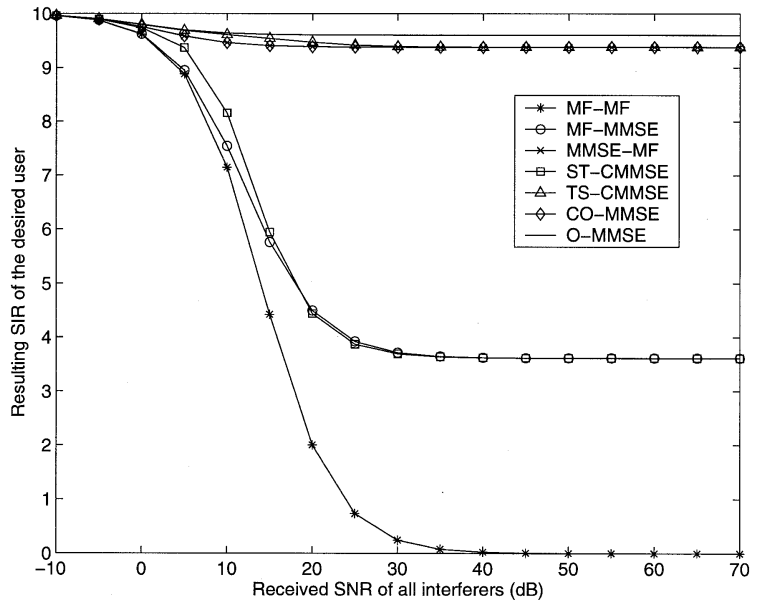

Fig. 2. $\quad N=2, K=2, G=8$.

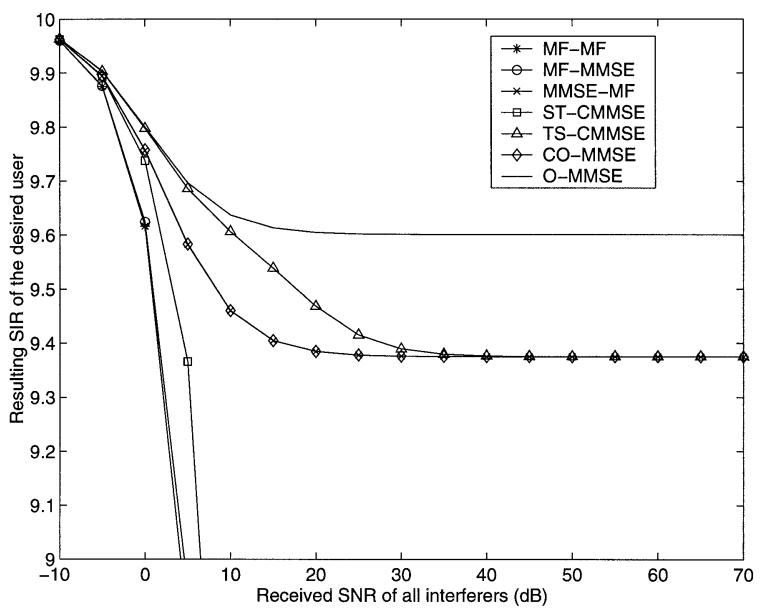

Fig. 3. $N=2, K=2, G=8$, Fig. 2 magnified.

becomes interference limited, both detectors have identical performance. The constrained optimum MMSE filter outperforms all other filters, except for the optimum MMSE filter, which is the SIR maximizer among all matrix filters.

For the rest of this section, the system considered has $K=4$ antenna array elements and $G=16$ processing gain. We will examine the performance of the filter structures for this system under different loading conditions.

We first consider $N=8$ users. Fig. 4 shows the output SIR of the desired user. The constrained optimum MMSE filter outperforms all filters, except for the optimum MMSE filter. Note that, for this system, users' temporal signature sequences are linearly independent. Thus, when the system is interference limited, the filters that perform temporal MMSE first, i.e., the temporal MMSE filter-spatial matched filter of Section III-C and the cascaded temporal-spatial MMSE filter of Section III-D, end up decorrelating all interferers in the temporal domain. Specifically, the temporal MMSE filter at the output of each antenna becomes a decorrelator $\mathbf{c}_{k}=\mathbf{c}$, for all $k=1, \ldots, K$, for the the cascaded temporal-spatial MMSE filter. In this case, the

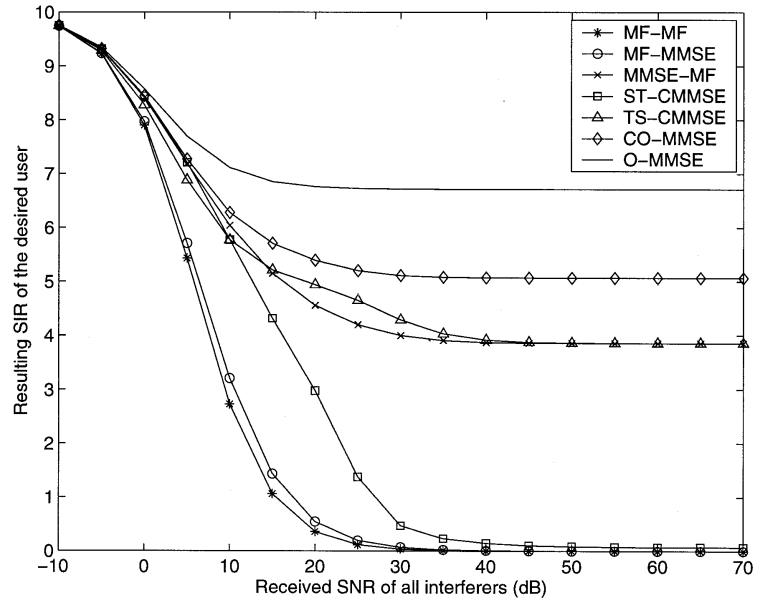

Fig. 4. $N=8, K=4, G=16$.

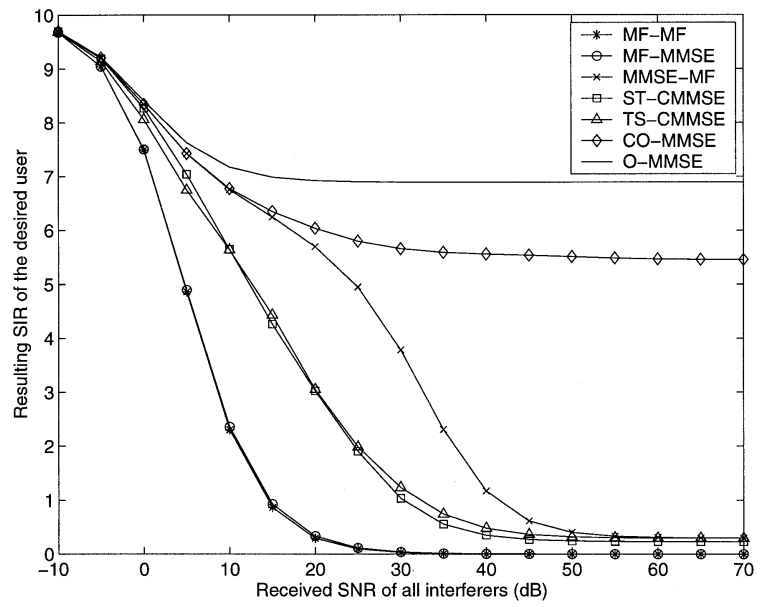

Fig. 5. $\quad N=16, K=4, G=16$.

output statistics of the first stage of the cascaded temporal-spatial MMSE filter are interference free, i.e., (14) can be expressed as

$$
\tilde{\mathbf{y}}=\sqrt{p_{i} h_{i}} b_{i}\left(\mathbf{c}^{H} \mathbf{s}_{i}\right) \mathbf{a}_{i}+\tilde{\mathbf{n}}
$$

where $\tilde{n}_{k}$ is the enhanced noise at the output of the $k$ th antenna and the components of $\tilde{\mathbf{n}}$ are independent. Thus, the second-stage spatial MMSE combiner $\mathbf{w}$ in (16) becomes the spatial matched filter $\mathbf{a}_{i}$, which explains why the temporal MMSE-spatial matched filter and the cascaded temporal-spatial MMSE filter have identical performance asymptotically. It is also notable that a constrained optimum MMSE detector becomes a temporal-spatial decorrelator and chooses to suppress some of the interferers in temporal domain and others in spatial domain, such that it gets the best asymptotic SIR among such temporal-spatial decorrelators.

Next we consider $N=16$ users. The output SIR of the desired user is plotted in Fig. 5. There are still enough temporal dimensions for users to be decorrelated in the temporal domain, i.e., $N \leq G$, even if they cannot all be suppressed in the spatial domain. As a result, all filters that employ MMSE combining in the temporal domain have nonzero SIRs asymptotically, i.e., $\mathbf{X}_{i}^{\mathrm{MMSE}-\mathrm{MF}}, \mathbf{X}_{i}^{\mathrm{ST}-\mathrm{CMMSE}}, \mathbf{X}_{i}^{\mathrm{TS}-\mathrm{CMMSE}}, \mathbf{X}_{i}^{\mathrm{CO}-\mathrm{MMSE}}$, and 


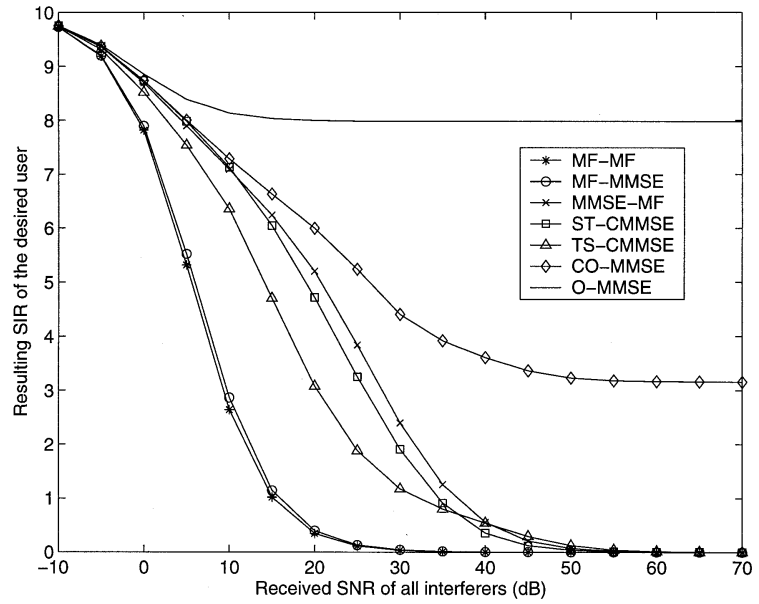

Fig. 6. $N=18, K=4, G=16$.

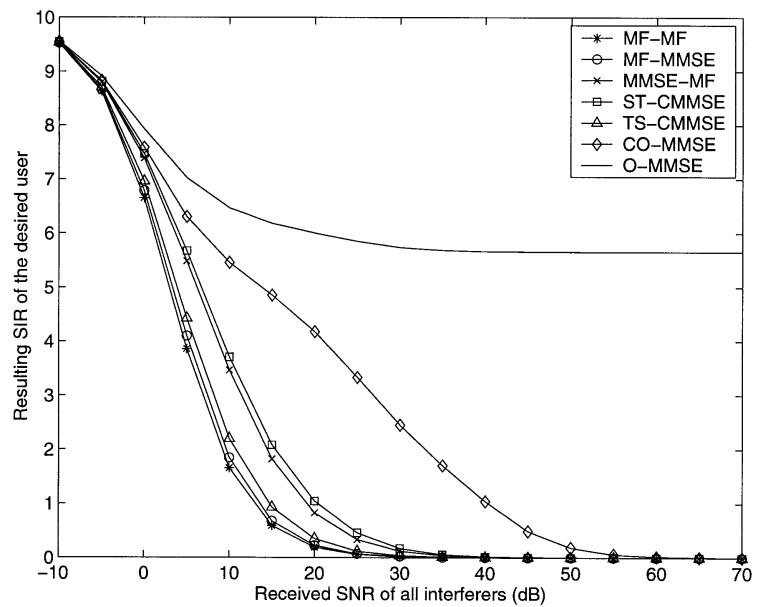

Fig. 7. $N=36, K=4, G=16$.

$\mathbf{X}_{i}^{\mathrm{O}-\mathrm{MMSE}}$ are all near-far resistant. However, the constrained optimum MMSE filter, again by choosing the appropriate users to suppress in the spatial or temporal domains, achieve higher SIR over all filters except the optimum MMSE filter.

The next system to be considered has $N=18$ users, and results are shown in Fig. 6. Since the number of interferers is larger than both the processing gain and the number of array elements, all interferers cannot be suppressed in a single domain; thus the combined single-user/multiple-user filter structures, i.e., $\mathbf{X}_{i}^{\mathrm{MMSE}-\mathrm{MF}}$ and $\mathbf{X}_{i}^{\mathrm{MF}-\mathrm{MMSE}}$, are not near-far resistant. For this example, the cascaded structures, i.e., $\mathbf{X}_{i}^{\mathrm{ST}-\mathrm{CMMSE}}$ and $\mathbf{X}_{i}^{\mathrm{TS}-\mathrm{CMMSE}}$, are not near-far resistant either, since in the interference-dominated regime, each stage tries to suppress all interferers independently in cascade. When an interference suppressor is designed by considering both domains jointly, as in the case of constrained optimum and optimum detectors, $\mathbf{X}_{i}^{\mathrm{CO}-\mathrm{MMSE}}$ and $\mathbf{X}_{i}^{\mathrm{O}-\mathrm{MMSE}}$, near-far resistance is achieved.

The last example we consider is a very highly loaded system with $N=36$ users. The purpose of this experiment is to show the difference between the optimum MMSE filter and the constrained optimum MMSE filter. We observe from Fig. 7 that although the constrained optimum MMSE filter $\mathbf{X}_{i}^{\mathrm{CO}-\mathrm{MMSE}}$ results in acceptable SIR values in near-far situations, e.g., an output SIR of $5(7 \mathrm{~dB})$ when all interferers' powers are $10 \mathrm{~dB}$ higher than the desired user, it is not near-far resistant. This is simply due to the fact that the constrained optimum MMSE filter can suppress up to $G-1$ users in the temporal domain and $K-1$ users in the spatial domain. Thus, for this example, when $N>19$, the constrained optimum MMSE filter is not able to suppress all the interference and the output SIR it produces approaches zero when the interferers' powers approach infinity. The optimum MMSE detector, on the other hand, can suppress up to $K G-1$ users and for this example is near-far resistant.

\section{CONCLUSION}

In this paper, we have examined different possible filter structures for a CDMA system when both temporal and spatial filters are used at the receiver. It is shown that the joint domain approaches, the optimum MMSE filter and the constrained optimum MMSE filter, outperform the approaches where the temporal and spatial receivers are designed independently in cascade. While the optimum MMSE is the filter that maximizes the output SIR of the desired user over all possible temporalspatial filter structures, its complexity may render its usage impractical, and other structures have to be considered. We have observed that the constrained optimum MMSE filter performs close to the optimum MMSE filter for all but extremely loaded systems, and outperforms all other structures either uniformly or asymptotically (in the decorrelating regime), and shows promise for implementability because of its low complexity.

\section{REFERENCES}

[1] F. Adachi, M. Sawahashi, and H. Suda, "Wideband DS-CDMA for nextgeneration mobile communication systems," IEEE Commun. Mag., vol. 36, pp. 56-69, Sept. 1998.

[2] E. Dahlman, B. Gudmundson, M. Nilsson, and J. Skold, "UMTS/IMT-2000 based on wideband CDMA," IEEE Commun. Mag., vol. 36, pp. 70-80, Sept. 1998.

[3] E. Dahlman, P. Beming, F. Ovesjo, J. Knutsson, M. Persson, and C. Roobol, "WCDMA - The radio interface for future mobile multimedia communications," IEEE Trans. Veh. Technol., vol. 47, pp. 1105-1118, Nov. 1998.

[4] J. Zander, "Performance of optimum transmitter power control in cellular radio systems," IEEE Trans. Veh. Technol., vol. 41, pp. 57-62, Feb. 1992.

[5] S. Verdú, Multiuser Detection. Cambridge, U.K.: Cambridge Univ. Press, 1998.

[6] A. F. Naguib, A. J. Paulraj, and T. Kailath, "Capacity improvement with base-station antenna arrays in cellular CDMA," IEEE Trans. Veh. Technol., vol. 43, pp. 691-698, Aug. 1994.

[7] S. Ulukus and R. D. Yates, "Iterative signature adaptation for capacity maximization of CDMA systems," presented at the 36th Annual Allerton Conf. Communications, Control and Computing, Sept. 1998.

[8] F. Rashid-Farrokhi, K. J. R. Liu, and L. Tassiulas, "Transmit beamforming for cellular communication systems," in Conf. Information Sciences and Systems, 1997, pp. 92-97.

[9] S. Verdú, "Computational complexity of multiuser detection," Algorithmica, vol. 4, no. 4, pp. 303-312, 1989.

[10] R. Lupas and S. Verdú, "Linear multiuser detectors for synchronous code-division multiple-access channels," IEEE Trans. Inform. Theory, vol. 35, pp. 123-136, Jan. 1989.

[11] U. Madhow and M. L. Honig, "MMSE interference suppression for direct-sequence spread-spectrum CDMA," IEEE Trans. Commun., vol. 42, pp. 3178-3188, Dec. 1994.

[12] A. Duel-Hallen, "Decorrelating decision-feedback multiuser detector for synchronous code-division multiple-access channels," IEEE Trans. Commun., vol. 41, pp. 285-290, Feb. 1993.

[13] A. J. Paulraj and C. B. Papadias, "Space-time processing for wireless communications,” IEEE Signal Processing Mag., pp. 49-83, Nov. 1997. 
[14] X. Bernstein and A. M. Haimovich, "Space-time optimum combining for CDMA communications," Wireless Personal Commun., no. 3, pp. 73-89, 1996.

[15] R. Kohno, H. Imai, M. Hatori, and S. Pasupathy, "Combination of an adaptive array antenna and a canceller of interference for direct-sequence spread-spectrum multiple-access system," IEEE J. Select. Areas Commun., vol. 8, pp. 675-682, May 1990.

[16] T. F. Wong, T. M. Lok, J. S. Lehnert, and M. D. Zoltowski, “A linear receiver for direct-sequence spread-spectrum multiple-access systems with antenna arrays and blind adaptation," IEEE Trans. Inform. Theory, vol. 44, pp. 659-676, Mar. 1998.

[17] X. Wang and H. V. Poor, "Space-time multiuser detection in multipath CDMA channels," IEEE Trans. Signal Processing, vol. 47, pp. 2356-2374, Sept. 1999

[18] A. Yener, R. D. Yates, and S. Ulukus, "Joint power control, multiuser detection and beamforming for CDMA systems," in IEEE Vehicular Technology Conf. (VTC'99), May 1999, pp. 1032-1036

[19] _ - "Interference management for CDMA systems through power control, multiuser detection, and beamforming," IEEE Trans. Commun., vol. 49, pp. 1227-1239, July 2001.

[20] R. A. Monzingo and T. W. Miller, Introduction to Adaptive Arrays. New York: Wiley, 1980

[21] V. G. Subramanian and U. Madhow, "Blind demodulation of direct-sequence CDMA signals using an antenna array," presented at the CISS'96, 1996

[22] M. Honig, U. Madhow, and S. Verdú, "Blind adaptive multiuser detection," IEEE Trans. Inform. Theory, vol. 41, pp. 944-960, July 1995.

[23] J. A. Fessler and A. O. Hero, "Space-alternating generalized expectation-maximization algorithm," IEEE Trans. Signal Processing, vol. 42, pp. 2664-2677, Oct. 1994.

[24] H. V. Poor and S. Verdú, "Probability of error in MMSE multiuser detection," IEEE Trans. Inform. Theory, vol. 43, pp. 858-871, May 1997.

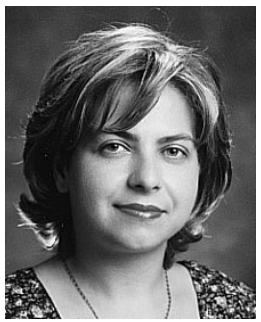

Aylin Yener (S'93-M'00) received the B.S. degree in electrical and electronics engineering and in physics from Boğaziçi University, Istanbul, Turkey, in 1991 and the M.S. and Ph.D. degrees in electrical and computer engineering from Rutgers-The State University, Piscataway, NJ, in 1994 and 2000, respectively.

During her Ph.D. studies, she was with the Wireless Information Network Laboratory (WINLAB), Department of Electrical and Computer Engineering, Rutgers-The State University. Between fall 2000 and fall 2001, she was with the Electrical Engineering and Computer Science Department, Lehigh University, Bethlehem, PA, where she was a P. C. Rossin Assistant Professor. Currently, she is with the Electrical Engineering Department, Pennsylvania State University, University Park, as an Assistant Professor. Her research interests include performance enhancement of CDMA systems, wireless communication theory, and wireless networking in general.

Dr. Yener is an Associate Editor of the IEEE TRANSACTIONS ON WIRELESS COMMUNICATIONS.

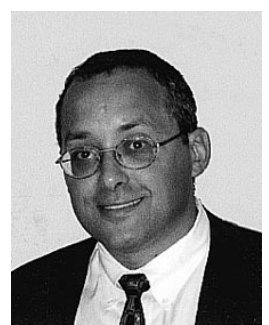

Roy D. Yates (M'91) received the B.S.E. degree from Princeton University, Princeton, NJ, in 1983 and the S.M. and Ph.D. degrees from the Massachsuetts Institute of Technology, Cambridge, in 1986 and 1990, respectively, all in electrical engineering.

Since 1990, he has been with the Wireless Information Networks Laboratory (WINLAB) and the Electrical and Computer Engineering (ECE) Department, Rutgers-The State University, Piscataway, NJ. Presently, he is the associate director of WINLAB and a Professor in the ECE Department. He is a coauthor (with D. Goodman) of Probability and Stochastic Processes: A Friendly Introduction for Electrical and Computer Engineers (New York: Wiley, 1999). His research interests include power control, interference suppression, and media access protocols for wireless communications systems.

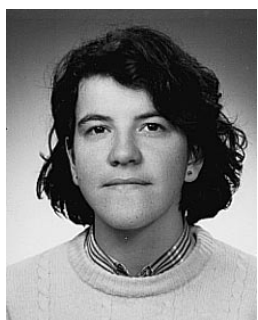

Sennur Ulukus (S'92-M'98) received the B.S. and M.S. degrees in electrical and electronics engineering from Bilkent University, Ankara, Turkey, in 1991 and 1993, respectively, and the Ph.D. degree in electrical and computer engineering from Rutgers-The State University, Piscataway, NJ, in 1998.

During her Ph.D. studies, she was with the Wireless Information Network Laboratory (WINLAB), Rutgers-The State University. From 1998 to 2001, she was a Senior Technical Staff Member at AT\&T Labs-Research, NJ. Since August 2001, she has been an Assistant Professor in the Department of Electrical and Computer Engineering, University of Maryland at College Park, where she also holds a joint appointment with the Institute for Systems Research. Her research interests are in wireless communication theory and networking, communication theory, and information theory. 